\title{
CARACTERÍSTICAS FÍSICAS DE FRUTOS DE CUACHALALATE (Amphipterygium adstringens (Schltdl.) Standl) DE TRES PROCEDENCIAS
}

\author{
PHYSICAL CHARACTERISTICS OF CUACHALALATE (Amphipterygium adstringens \\ (Schltdl.) Standl.) FRUITS FROM THREE PROVENANCES
}

\author{
Areli M. Guzmán-Pozos y Efraín Cruz-Cruz
}

Campo Experimental Zacatepec, Instituto Nacional de Investigaciones Forestales, Agrícolas y Pecuarias. Km 0.5 Carr. Zacatepec-Galeana, Col. Centro. 62780, Zacatepec, Morelos, México. Tel 017343430368 y 017343431246.

*Autor para correspondencia (cruz.efrain@inifap.gob.mx)

\section{RESUMEN}

El fruto de cuachalalate (Amphipterygium adstringens (Schltdl.) Standl.) es una sámara, seco e indehiscente, con presencia de una ala aplanada fundida con la semilla. La corteza de esta especie se utiliza en la medicina tradicional para el tratamiento de diversas enfermedades. El presente estudio tuvo como objetivo conocer la variación morfológica del fruto de cuachalalate de tres procedencias, dos del Estado de Morelos y una del Estado de Oaxaca. Las procedencias fueron San Juan de los Cués, Oaxaca, Quilamula, Morelos y Zacatepec, Morelos. Se registraron las variables de largo del fruto y ala; largo, ancho y grosor del área seminífera, así como número de semillas y de lóculos por fruto. Para el primer grupo de variables, los valores se registraron en dos muestras de 30 frutos por árbol tomados en forma aleatoria en San Juan de los Cués y Quilamula (20 individuos por procedencia) y en Zacatepec (18 individuos). El número de semillas y lóculos se determinó con muestras aleatorias de 30 frutos con cuatro repeticiones por árbol, con un total de 6960 frutos cortados. Los datos se analizaron con un diseño experimental de parcelas divididas, donde la parcela grande fueron las procedencias y la parcela chica los árboles. Se encontraron diferencias significativas $(P=0.01)$ en las procedencias para las variables: largo del fruto $(52.0 \pm 5.0,43.9 \pm 3.6$ y $39.0 \pm 3.5 \mathrm{~mm})$; largo del ala $(38.4 \pm$ $4.0,31.0 \pm 3.2$ y $27.6 \pm 3.3 \mathrm{~mm})$; largo $(13.6 \pm 1.2,12.8 \pm 1.1$ y $11.3 \pm 0.9$ $\mathrm{mm})$, ancho $(17.6 \pm 1.8,17.5 \pm 1.8$ y $16.6 \pm 1.9 \mathrm{~mm})$ y grosor $(10.5 \pm$ $0.8,9.9 \pm 0.8$ y $9.1 \pm 0.6 \mathrm{~mm}$ ) del área seminífera, para las procedencias Zacatepec, Quilamula y San Juan de los Cués, respectivamente. Se concluyó que el cuachalalate presenta variación morfológica del fruto y los porcentajes de fruto con una semilla fueron $43.2 \pm 10.3 \%, 32.3 \pm 13.1$ $\%$ y $24.6 \pm 12.6 \%$ para Zacatepec, Quilamula y San Juan de los Cués.

Palabras clave: Amphipterygium adstringens, fruto, procedencia, sámara, variabilidad.

\section{SUMMARY}

The cuachalalate (Amphipterygium adstringens (Schltdl.) Standl.) fruit is a dry and indehiscent samara with a flattened wing. Its bark is used in traditional medicine against many diseases. This research studied morphological variation of the fruit from three provenances of cuachalalate: San Juan de los Cués, Oaxaca State, Quilamula, Morelos State and Zacatepec, Morelos State. Fruit and wing length; and length, width and thickness of pericarp were recorded. Two samples of 30 fruits per tree were randomly taken in San Juan de los Cués and Quilamula (20 individuals) and Zacatepec (18 individuals). The number of seeds and locules per fruit was recorded with random samples of 30 fruits and four replicates per tree. The data was analyzed as a split plot experimental design, where the main plot was the provenance and the subplots were the trees. Significant differences $(P=0.01)$ were found for the variables: fruit length $52.0 \pm 5.0,43.9 \pm 3.6$ and $39.0 \pm 3.5$ $\mathrm{mm}$, wing length $38.4 \pm 4.0,31.0 \pm 3.2$ and $27.6 \pm 3.3 \mathrm{~mm}$; length 13.6 $\pm 1.2,12.8 \pm 1.1$ and $11.3 \pm 0.9 \mathrm{~mm}$, width $17.6 \pm 1.8,17.5 \pm 1.8$ and $16.6 \pm 1.9 \mathrm{~mm}$, and pericarp thickness $10.5 \pm 0.8,9.9 \pm 0.8$ and $9.1 \pm 0.6$ $\mathrm{mm}$, for Zacatepec, Quilamula and San Juan de los Cués, respectively. Percentage of fruit with one seed was $43.2 \pm 10.3 \%, 32.3 \pm 13.1 \%$ and 24.6 $\pm 12.6 \%$ from Zacatepec, Quilamula and San Juan de los Cués, respectively. It was concluded that cuachalalate has morphological fruit variation.

Index words: Amphipterygium adstringens, fruit, provenance, samara, variability.

\section{INTRODUCCIÓN}

Amphipterygium adstringens (Schltdl.) Standl.) es un árbol endémico de México que crece en la selva baja caducifolia donde es dominante, y generalmente se asocia con diversas especies de Bursera y Pseudosmodingium perniciosum (Kunth) Engl. (Pennington y Sarukhán, 2005). A la planta se le conoce como palo de rosa, cuachalalá, cuachalalate, maxiterán y volador (García, 2006). La distribución del cuachalalate se restringe en la vertiente del Pacífico, en los Estados de Sinaloa, Nayarit, Jalisco, Colima, Michoacán, Morelos, Guerrero, Oaxaca y Chiapas (Solares, 2002).

Por el cambio de uso del suelo, las poblaciones de la especie se han reducido y en algunos casos perdido, y con ello disminuido la variabilidad genética de sus individuos (Solares et al., 2006). Actualmente, el aprovechamiento de la corteza de $A$. adstringens es intensivo y destructivo por la forma tradicional de recolecta, ya que se afecta al cámbium y al floema y por ello ocasiona la muerte de los árboles; esto pone en riesgo la estabilidad de las poblaciones naturales y la conservación de la especie (Solares y Gálvez, 2002). Aunado a lo anterior, las semillas presentan bajos porcentajes de germinación, debido al reducido número de semillas formadas y viables por fruto (Cid, 2008). 
El cuachalalate es una de las especies de mayor relevancia en la herbolaria mexicana, debido a que cura más de 30 enfermedades (Solares et al., 2006; Solares et al., 2012). La corteza se usa para endurecer encías, lavar heridas y para el tratamiento del cáncer de estómago (García, 2006). Por esta razón se han aislado e identificado sus compuestos activos como triterpenos (ácidos masticadienoico, hidroximasticadienoico) y mezclas de ácidos masticadienoicos en corteza de plantas del sexo femenino (García, 2006).

El cuachalalate, el cirián (Crescentia alata Kunth) y el ciruelo (Spondias mombin L.) son especies demandadas por su uso medicinal y artesanal, y por su alto grado de comercialización el cuachalalate se ubica entre las especies amenazadas en los Estados de Morelos, Guerrero y Oaxaca (Boyás et al., 2001). Por ello es conveniente repoblar con esta especie los ambientes de la selva baja caducifolia (Monroy, 2010). Además de su importancia ecológica, el cuachalalate contribuye en la conservación de la guacamaya verde (Ara militaris L.) debido a que ésta se alimenta del fruto y de las hojas del árbol (Medina, 2000; Bonilla et al., 2008).

A. adstringens pertenece a la familia Julianiaceae y se relaciona con la Burseraceae debido a su hábitat y el follaje de los árboles; y con la Anacardiaceae, especialmente con el género Rhus, por la similitud que existe en la anatomía de la madera y por las sustancias químicas presentes, como los flavonoides (Cuevas, 2005). El cuachalalate es dioico y presenta frutos colgantes en racimo (Cuevas, 2005; Pennington y Sarukhán, 2005). Su fruto es una sámara, seco, indehiscente y fibroso, que se encuentra sobre pedicelos aplanados y acrescentes hasta formar una especie de ala de $3 \mathrm{a} 4 \mathrm{~cm}$, y en cada fruto hay de una o dos semillas aplanadas de $5 \mathrm{~mm}$ de largo (García-Fayos et al., 2001; Pennington y Sarukhán, 2005). La floración se presenta de junio a agosto y la fructificación de agosto a enero (Cuevas, 2005). En relación con la variación en el tamaño y forma del fruto, se tiene la referencia que una misma especie presenta variabilidad debido a la presencia de genes que los hacen únicos (White, 2002; Franco-Mora et al., 2010), y por las características ambientales donde se desarrollan los árboles (Iglesias et al., 2006).

Según Mostacedo et al. (2001), el fruto que presenta ala desempeña una doble función, ya que protege a las semillas y asegura su dispersión por el viento (anemocoria) y establecimiento en un claro del bosque. Esta dispersión ocurre principalmente al final de la época seca, y por la característica que posee el fruto es capaz de flotar y así se dispersa secundariamente a lo largo de las corrientes de agua (GarcíaFayos et al., 2001).

Para conservar la especie es importante conocer las características del fruto, ya que éstas se encuentran relacio- nadas con las semillas y con la germinación (Flores, 2002; Arteaga, 2007). En el caso de cuachalalate existe limitada información, por lo que es necesario generar más conocimiento que contribuya a restaurar las poblaciones naturales, y disponer de información para el establecimiento de plantaciones comerciales para disminuir la presión sobre las poblaciones naturales.

El objetivo de este trabajo fue conocer la variación morfológica del fruto de cuachalalate de tres procedencias, dos de ellas del Estado de Morelos y una del Estado de Oaxaca.

\section{MATERIALES Y MÉTODOS}

El fruto de cuachalalate utilizado provino de tres procedencias: San Juan de los Cués (Oaxaca), Quilamula (Morelos) y Zacatepec (Morelos). La recolecta se hizo en noviembre del 2010. Los sitios de colecta de San Juan de los Cués y Quilamula se ubicaron con recorridos de campo y con autorización de los pobladores, y en Zacatepec la colecta fue de la plantación de 11 años ubicada en el Campo Experimental Zacatepec del Instituto Nacional de Investigaciones Forestales, Agrícolas y Pecuarias. Los árboles se seleccionaron con base en sus características fenotípicas. El color café y la textura fibrosa y papirácea de la cubierta del fruto se consideraron como indicador de madurez, además de su fácil desprendimiento de las ramas.

En árboles altos los frutos se recolectaron trepando a la copa y en ocasiones con ayuda de un gancho para desprender los frutos, los cuales se depositaron en tenates (recipientes de palma para facilitar el manejo) y se guardaron en bolsas de papel Kraft ${ }^{\circledR}$. Para evitar la mezcla de las muestras, la bolsa se etiquetó con la información de la procedencia: fecha, sitio y municipio de colecta.

De las procedencias de San Juan de los Cués (Oaxaca) y Quilamula (Morelos) se tomaron muestras de 20 árboles, y para evitar problemas de parentesco se consideró una distancia mínima de $100 \mathrm{~m}$ entre los árboles seleccionados, como recomienda Willan (1991). En la plantación ubicada en Zacatepec se recolectaron muestras de 18 árboles. A los árboles seleccionados se les midió altura total, diámetro del fuste y diámetro de la copa. De cada procedencia se registró la altitud y las coordenadas geográficas (Cuadro 1).

El beneficio del fruto se realizó en el Laboratorio de Semillas del Campo Experimental Zacatepec, Morelos. El fruto se colocó sobre papel periódico para su secado bajo sombra, y la limpieza consistió en la separación de restos de hojas y ramas. Para conocer la variabilidad en el tamaño del fruto de los diferentes árboles y de las tres procedencias, se midió el largo del fruto y ala; y largo, ancho y grosor del área seminífera. La medición se hizo con 
Cuadro 1. Ubicación geográfica y características ambientales de los sitios de recolecta de las procedencias de $A$. adstringens.

\begin{tabular}{|c|c|c|c|c|c|}
\hline \multirow{2}{*}{ Procedencias } & \multicolumn{2}{|c|}{ Ubicación geográfica } & \multirow{2}{*}{ Altitud (m) } & \multirow{2}{*}{$\begin{array}{c}\text { Precipitación } \\
\mathrm{mm}\end{array}$} & \multirow{2}{*}{$\begin{array}{c}\text { Temperatura } \\
{ }^{\circ} \mathrm{C}\end{array}$} \\
\hline & $\mathrm{N}$ & $\mathrm{O}$ & & & \\
\hline San Juan de los Cués, Oax. & $18^{\circ} 02^{\prime} 58.5^{\prime \prime}$ & $97^{\circ} 05^{\prime} 13.5^{\prime \prime}$ & 885 & 848 & 29.2 \\
\hline Quilamula, Mor. & $18^{\circ} 31^{\prime} 31.8^{\prime \prime}$ & $99^{\circ} 03^{\prime} 06.8^{\prime \prime}$ & 1089 & 958.6 & 31.9 \\
\hline Zacatepec, Mor. & $18^{\circ} 37^{\prime} 30.0^{\prime \prime}$ & $99^{\circ} 14^{\prime} 05.3^{\prime \prime}$ & 910 & 1060.4 & 32.3 \\
\hline
\end{tabular}

un vernier digital marca Mitutoyo ${ }^{\circledR}$ (Kawasaki, Japón). El tamaño de la unidad experimental fue de 30 frutos tomados al azar y dos repeticiones por árbol, para un total de 3480 frutos.

Para determinar el número de semillas y de lóculos se tomaron muestras aleatorias de 30 frutos con cuatro repeticiones por árbol, de los muestreados de cada procedencia; en total se cortaron 6960 frutos. El número de lóculos y las semillas completas formadas se contaron en frutos previamente cortados en forma transversal, a la mitad de la parte seminífera.

El análisis estadístico se hizo con un diseño de parcelas divididas, en el que la parcela grande correspondió a las procedencias y la parcela chica a los árboles, con el siguiente modelo propuesto por Juárez-Agis et al. (2006):

$$
Y_{i k j}=\mu+P_{j}+A(P)_{k j}+E_{i k j}
$$

$Y_{i k j}=$ valor observado de la $i$-ésima variable del $k$-ésimo árbol, en la j-ésima procedencia; $\mu=$ media poblacional; $P_{j}=$ efecto aleatorio de la j-ésima procedencia; $A(P)_{k j}=$ efecto aleatorio del $k$-ésimo árbol dentro de la j-ésima procedencia; $E_{i k j}=$ error de muestreo.

A los datos se les hizo análisis de varianza con el paquete estadístico SAS para Windows Versión 8.1 (SAS, 19992000). Para la comparación de medias se utilizó la prueba de Tukey $(\alpha=0.05)$. Adicionalmente se hizo el análisis de correlación de Pearson entre las variables registradas del fruto (Castillo, 2007).

\section{RESULTADOS Y DISCUSIÓN}

\section{Diferencias entre procedencias}

Los análisis de varianza mostraron diferencias significativas $(P=0.0001)$ entre procedencias en el largo del fruto y ala, largo, ancho y grosor del área seminífera. La procedencia de San Juan de los Cués presentó los frutos más pequeños $(39.0 \pm 3.5 \mathrm{~mm})$ y la de Zacatepec los frutos más grandes $(52.0 \pm 5.0 \mathrm{~mm})$ (Cuadro 2). Esta variación entre procedencias se atribuye a que la especie establece una relación particular con su ambiente (Piedrahita, 1997) y a su adaptación para sobrevivir en las condiciones ambientales y ecológicas en los sitios donde se desarrolla (Cabrera, 2006). Como se ha confirmado en el género Pinus (Iglesias et al., 2006) y en las especies Phaseolus lunatus L. (Vargas et al., 2003), Guazuma ulmifolia Lambert y Quercus oleoides Schl. Et Cham. (Márquez et al., 2005).

La adaptación fisiológica de una especie es una respuesta a las condiciones ambientales particulares en las que se encuentran los individuos; esta adaptación se observa en la variabilidad morfológica del fruto y de la semilla (Gómez et al., 2010). Existen otros casos donde las poblaciones muestran uniformidad en la características del fruto, aun en poblaciones silvestres, lo que permite un aislamiento entre poblaciones, como lo encontrado en Ricinus communis L. (Mazzani y Rodríguez, 2009). La variación entre poblaciones también se asocia con las barreras geográficas que limitan el flujo de genes entre ellas (Zamora, 2002).

El flujo de genes de la población de cuachalalate de San Juan de los Cués es limitado por la ubicación geográfica de la localidad en la región de la Cañada del Estado de Oaxaca. Esta región está rodeada por la Sierra Norte de Oaxaca y la Mixteca oaxaqueña en la parte sur y noroeste, y por el norte por el Valle de Tehuacán, Puebla.

\section{Diferencias dentro de procedencias}

Se encontraron diferencias significativas $(P=0.0001)$ entre árboles dentro de procedencia. Los valores mínimos y máximos para largo del fruto en San Juan de los Cués fueron 33.9 y $50.1 \mathrm{~mm}$; en Quilamula, 38 y $52 \mathrm{~mm}$; y en Zacatepec, 44.5 y $62.3 \mathrm{~mm}$. Para largo del ala, en San Juan de los Cués los valores fueron 23.2 y $37.6 \mathrm{~mm}$; en Quilamula, 25.6 y $37.9 \mathrm{~mm}$; y en Zacatepec 32.1 y $46.3 \mathrm{~mm}$.

Los valores medios del largo del fruto y del ala de la procedencia de Zacatepec se localizaron por arriba de las medias de los tres sitios, mientras que la ubicación de los valores de San Juan de los Cués y Quilamula se ubicaron debajo de dichas medias (Figuras 1 y 2 ).

Los mayores valores de las variables registradas del fruto en la procedencia de Zacatepec se atribuyen al manejo inicial 
Cuadro 2. Comparación de medias de cinco características físicas de los frutos de A. adstringens de tres procedencias (Valor medio \pm desviación estándar).

\begin{tabular}{|c|c|c|c|c|c|}
\hline \multirow{2}{*}{ Procedencia } & \multirow{2}{*}{$\begin{array}{l}\text { Largo de fruto } \\
\qquad(\mathrm{mm})\end{array}$} & \multirow{2}{*}{$\begin{array}{l}\text { Largo del ala } \\
\quad(\mathrm{mm})\end{array}$} & \multicolumn{3}{|c|}{ Área seminífera } \\
\hline & & & Largo (mm) & Ancho $(\mathrm{mm})$ & Grosor $(\mathrm{mm})$ \\
\hline SJC & $39.0 \pm 3.5 c$ & $27.6 \pm 3.3 c$ & $11.3 \pm 0.9 \mathrm{c}$ & $17.5 \pm 1.9 \mathrm{~b}$ & $9.1 \pm 0.6 c$ \\
\hline QM & $43.9 \pm 3.6 \mathrm{~b}$ & $31.0 \pm 3.2 \mathrm{~b}$ & $12.8 \pm 1.1 \mathrm{~b}$ & $16.6 \pm 1.8 \mathrm{a}$ & $9.9 \pm 0.8 \mathrm{~b}$ \\
\hline $\mathrm{ZC}$ & $52.0 \pm 5.0 \mathrm{a}$ & $38.4 \pm 4.0 \mathrm{a}$ & $13.6 \pm 1.2 \mathrm{a}$ & $17.5 \pm 1.8 \mathrm{a}$ & $10.5 \pm 0.8 \mathrm{a}$ \\
\hline
\end{tabular}

Medias con letras iguales no son estadísticamente diferentes (Tukey, 0.05); SJC = San Juan de los Cués, Oaxaca; QM = Quilamula, Morelos; ZC = Zacatepec, Morelos.

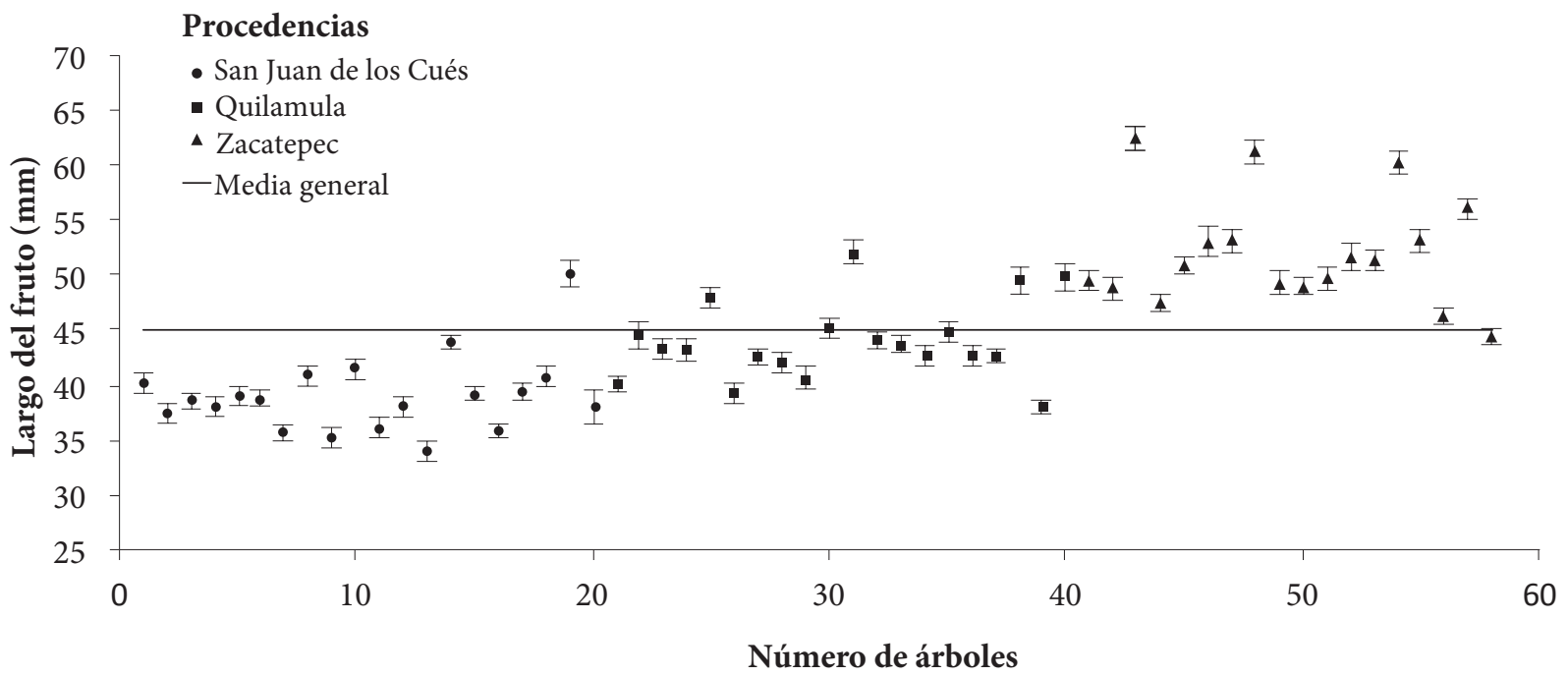

Figura 1. Largo promedio del fruto de 58 árboles de A. adstringens de tres procedencias (Intervalo de confianza $95 \%$ de probabilidad).

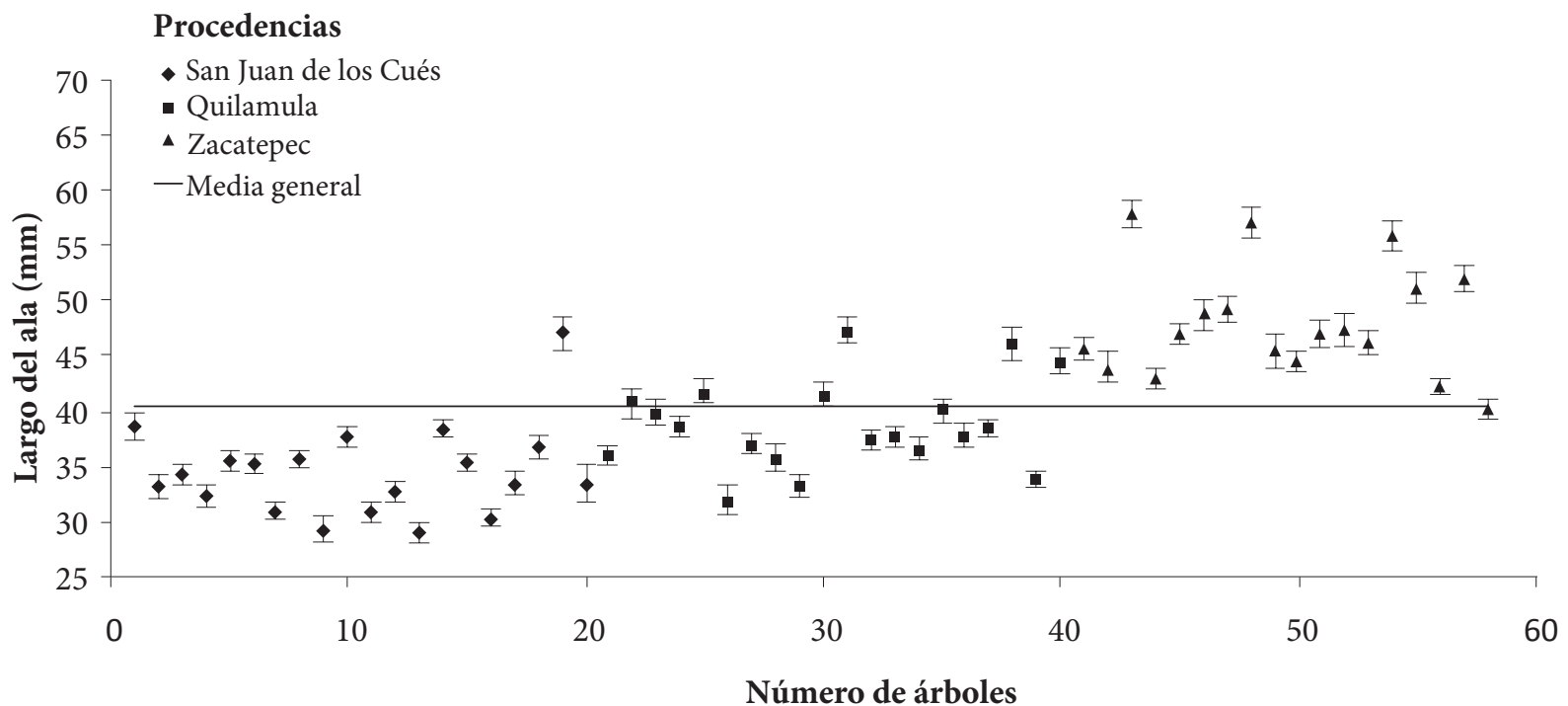

Figura 2. Largo promedio del ala de 58 árboles de A. adstringens de tres procedencias (Intervalo de confianza $95 \%$ de probabilidad). 
que se le dio a la plantación (riego, fertilización y podas) y a las condiciones edafoclimáticas donde se encuentran las plantas (suelos profundos, arcillosos y la precipitación más alta en relación con los otros dos sitios de recolecta); en cambio, las otras dos procedencias se encuentran en condiciones silvestres con pendientes, suelos pedregosos y arenosos, y baja precipitación (Cuadro 1).

El crecimiento del fruto se relaciona con la humedad disponible, como lo registrado en Teobroma cacao L. (Daymond y Hadley, 2008). Según Zavala (2004), los frutos de las especies que se encuentran en ambientes secos tienden a crecer más por la noche; adicionalmente, una mayor temperatura y poca humedad disponible influyen en el crecimiento más pequeño de los frutos, como lo encontrado en los frutos de San Juan de los Cués y de Quilamula. Según Houle et al. (2007), la variación en el tamaño del fruto se ve influenciada por su posición en el árbol, ya que los frutos grandes se encuentran en la parte superior de la copa por la mayor disponibilidad de azúcares y proteínas producidas por el área fotosintética, en comparación con los que se encuentran en la parte inferior del dosel.

La variación del largo y grosor del fruto de cuachalalate se relacionó de manera positiva con el número de semillas y número de lóculos. Las procedencias que resultaron con altos porcentajes de fruto sin semilla fueron San Juan de los Cués $(73.0 \pm 15.1 \%)$ y Quilamula $(60.4 \pm 17.6 \%)$, en comparación con la procedencia de Zacatepec $(26.0 \pm 13.1$ $\%)$. Los porcentajes de fruto con una semilla fueron de 24.6 $\pm 12.6 \%, 32.3 \pm 13.1 \%$ y $43.2 \pm 10.3 \%$; con dos semillas 2.8 $\pm 4.4 \%, 7.0 \pm 7.2 \%$ y $29.9 \pm 13.5 \%$, para las procedencias de San Juan de los Cués, Quilamula y Zacatepec, respectivamente. Los valores encontrados de fruto con tres o cuatro semillas fue por debajo de $1.1 \%$, para las tres procedencias. Se registró una variación en el número de lóculos dentro del fruto: la procedencia de San Juan de los Cués presentó dos lóculos con mayor frecuencia (50.0 $\pm 30.5 \%)$, en Quilamula la mayor frecuencia de frutos con tres lóculos (41.0 $\pm 18.3 \%)$, y en Zacatepec la mayor frecuencia con cuatro lóculos $(35.0 \pm 22.1 \%)$.

El fruto de mayor tamaño produce semillas con mejores características físicas. Esta afirmación fue corroborada en los frutos de cuachalalate por el número de semillas formadas. Según Niembro et al. (2007), el diámetro del fruto de Swietenia macrophylla King se encuentra relacionado positivamente con el número de semillas desarrolladas. El coeficiente de correlación entre el tamaño del fruto con el número de lóculos y semillas fue $r=0.78$ (no significativo) y $r=0.99(\mathrm{P}<0.05)$, lo que implica que a mayor tamaño del fruto se tendrá un mayor número se semillas potenciales y un mayor número de semillas formadas.
Las condiciones ambientales han favorecido la presencia de cuachalalate en los Estados de Guerrero, Puebla, Morelos y Oaxaca, que le permite crecer en hábitats específicos de la selva baja caducifolia (Solares, 2002). En esta amplia superficie que cubren los cuatro estados se presenta una gran variación orográfica que influye en las variables climáticas; estas diferencias ambientales determinan la adaptación de la especie (White, 2002; Iglesias et al., 2006; Franco-Mora et al., 2010). Esto se reflejó en las variaciones morfológicas del fruto de cuachalalate. Por ello se recomienda documentar la variabilidad genética de esta especie con la finalidad de conservar y fomentar las poblaciones naturales, determinar las mejores prácticas de manejo para su aprovechamiento y evitar el riesgo de extinción de la especie (Lobo y Medina, 2009).

La característica particular del fruto de cuachalalate, como el ala, es importante en la adaptación ecológica de la especie, ya que protege a las semillas de altas temperaturas y reduce su deshidratación en el interior del fruto y permite su dispersión a diferentes microhábitats por medio del viento; además, el fruto carece de aroma en comparación con frutos carnosos, debido a que su forma de dispersión es poco selectiva (Chacón y Bustamante, 2001).

\section{CONCLUSIONES}

Las procedencias evaluadas de Amphipterygium adstringens presentaron variación significativa en las variables de morfología del fruto: largo del fruto y ala; largo, ancho y grosor del área seminífera. Estas variables se presentaron en el siguiente orden: Zacatepec $>$ Quilamula $>$ San Juan de los Cués. Los valores máximos y mínimos del largo del fruto dentro de la procedencia fueron: en San Juan de los Cués, 33.9 y $50.1 \mathrm{~mm}$; en Quilamula, 38 y $52 \mathrm{~mm}$; y en Zacatepec, 44.5 y $62.3 \mathrm{~mm}$, respectivamente.

El cuachalalate presenta un alto porcentaje de frutos sin semilla, lo cual es fundamental conocer para la implementación de programas de reproducción sexual de la planta. Además es necesario considerar la procedencia del fruto porque el número de semillas por fruto es muy variable, como se encontró en el presente trabajo, donde la relación para el tamaño de fruto y número de semillas fue el siguiente: Zacatepec $>$ Quilamula $>$ San Juan de los Cués.

\section{BIBLIOGRAFÍA}

Arteaga L. L. (2007) El tamaño de las semillas de Vismia glaziovii Ruhl (Guttiferae) y su relación con la velocidad de germinación y tamaño de la plántula. Revista Peruana de Biología 14:17-20.

Bonilla R. C., R. Aguilar-S., R. García, R. Martínez y L. Cruz (2008) Monitoreo de la Población de la Guacamaya Verde en la Re serva de la Biosfera Tehuacán-Cuicatlán. Instituto Politécnico Nacional. Centro Interdisciplinario de Investigación para el Desarrollo Integral Regional-Oaxaca. México D. F. 105 p. 
Boyás D. J. C., M. A. Cervantes-S., J. M. Javelly-G., M. M. Linares-A., F. Solares-A., R. M. Soto-E., I. Naufal-T. y L. Sandoval-C (2001) Diagnóstico Forestal del Estado de Morelos. Secretaría de Agricultura, Ganadería, Desarrollo Rural, Pesca y Alimentación, Centro de Investigación Regional del Centro-Campo Experimental Zacatepec, Morelos, México. 181 p.

Cabrera E. I. E. (2006) Estudio de la Composición Arbórea, Fuente Semillera y Calidad de la Semilla de Caoba (Swietenia macrophylla King.) y Santa María (Calophyllum brasiliense var. Rekoi Standl.) en el Parque Nacional Laguna Lachuá, Cobán, Alta Verapaz. Universidad de San Carlos de Guatemala, Facultad de Agronomía. Guatemala. 66 p.

Castillo M. L. E. (2007) Introducción al SAS para Windows. 3ra ed. Universidad Autónoma de Chapingo. México. 295 p.

Chacón P. and R. O. Bustamante (2001) The effects of seed size and pericarp on seedling recruitment and biomass in Cryptocarya alba (Lauraceae) under two contrasting moisture regimes. Plant Ecology 152:137-144.

Cid de la T. K. S. (2008) Propagación Sexual de Cuachalalate (Amphipterygium adstringens), Especie de Uso Medicinal. Ed. Universidad Nacional Autónoma de México. México. 75 p.

Cuevas F. X. M. (2005) A revision of the genus Amphipterygium (Julianiaceae). Ibugana 13:27-47.

Daymond A. J. and P. Hadley (2008) Differential effects of temperature on fruit development and bean quality of contrasting genotypes of cacao (Teobroma cacao). Annals of Applied Biology 153:175-185.

Flores E. M. (2002) Seed biology. Tropical tree. In: Seed Manual. J. A. Vozzo (ed). United States Department of Agriculture. Forest Service y la Academia Nacional de Ciencias. Costa Rica. pp:13-118.

Franco-Mora O., S. Aguirre-Ortega, E. J. Morales-Rosales, A. González-Huerta y F. Gutiérrez-Rodríguez (2010) Caracterización morfológica y bioquímica de frutos de tejocote (Crataegus mexicana DC) de Lerma y Ocoyoacac, México. Ciencia Ergo Sum 17:61-66.

García G. K. Y. (2006) Principales usos y posibles acciones farmacológicas del cuachalalate (Amphipterygium adstringens Schiede ex Schltedl). InFÁRMAte 7:1-7.

García-Fayos P., J. Gulias, J. Martínez, A. Marzo, J. P. Melero, A. Traveset, $P$. Veintimilla, M. Verdú, V. Cerdán, M. Gasque y $\mathbf{H}$. Medrano (2001) Bases Ecológicas para la Recolección, Almacenamiento y Germinación de Semillas de Especies de uso Forestal de la Comunidad Valenciana. Banc de Llavors Forestals (Conselleria de Medi Ambient, Generalitat Valenciana). Valencia. $82 \mathrm{p}$.

Gómez J. D. M., C. Herrera R., J. Jasso M. y J. López Upton (2010) Variación en características reproductivas y germinación de semillas de Pinus leiophylla Schiede ex Schltdl. \& Cham. Revista Fitotecnia Mexicana 33:297-304.

Houle A., C. A. Chapman and W. L. Vickery (2007) Intratree variation in fruit production and implications for primate foraging. International Journal of Primatology 28:1197-1217.

Iglesias A. L. G., I. Mora y J. L. Casas-M (2006) Morfometría, viabilidad y variabilidad de las semillas de la población de Pinus hartwegii del Cofre de Perote Veracruz, México. Cuadernos de Biodiversidad 19:14-18.

Juárez-Agis A., J. López-Upton, J. J. Vargas-Hernández y C. Sáenz-Romero (2006) Variación geográfica en la germinación y crecimiento inicial de plántulas de Pseudotsuga menziesii de México. Agrociencia 40:783-792.

Lobo A. M. y C. I. Medina C. (2009) Conservación de recursos genéticos de la agrobiodiversidad como apoyo al desarrollo de sistemas de producción sostenibles. Revista Corpoica-Ciencia y Tecnología Agropecuaria 10:33-42.

Márquez R. J., L. C. Mendizábal H. y C. I. Flores R. (2005) Variación en semillas de Quercus oleoides Schl. Et. Cham. de tres poblaciones del centro de Veracruz, México. Foresta Veracruzana 7:31-36.

Mazzani E. y E. Rodríguez (2009) Estudio de la variabilidad presente en germoplasma de tártago (Ricinus communis L.) en cuanto a racimos, frutos y semillas. Revista UDO Agrícola 9:764-769.

Medina L. R. (2000) Julianiaceae Hemsl. Instituto de Biología, UNAM. Flora del Valle de Tehuacán-Cuicatlán 30:1-5

Monroy O. C. (2010) Conocimiento Ecológico Tradicional para la Gestión Sostenible de Especies Forestales no Maderables. Colegio de Postgraduados. Montecillo, México. 119 p.

Mostacedo B., M. Pereira y T. S. Frederiksen (2001) Dispersión de semillas anemócoras y autócoras durante la época seca en áreas con aprovechamiento forestal en un bosque seco tropical. Ecología en Bolivia 36:3-16.

Niembro R. A., E. O. Ramírez-G. y A. Aparicio-R. (2007) Correlación entre características de frutos de Swietenia Macrophylla King con su contenido de semilla desarrollada. Foresta Veracruzana 9:49-53.

Pennington D. T. y J. Sarukhán (2005) Árboles tropicales de México. In: Manual para la Identificación de las Principales Especies. 3ra ed. Fondo de Cultura Económica, México. 523 p.

Piedrahita C. E. (1997) Germinación de semillas de jacaranda copaia bajo condiciones contrastantes de luz. Crónica Forestal y del Medio Ambiente 12:1-4.

SAS Institute Inc (1999-2000) SAS User's Guide. Versión 8.1. North Carolina, U. S. A.

Solares A. F. (2002) Especies no maderables de clima tropical seco. Téc nicas de descortezamiento para hacer un uso sustentable de la corteza de cuachalalate (Amphipterygium adstringens Schiede ex Schlecht.) para su uso medicinal. SAGARPA. INIFAP. Folleto técnico No. 1. C. E. Zacatepec Mor., México. 2 p.

Solares A. F. y M. C. Gálvez-C. (2002) Manual para una Producción Sustentable de Corteza de Cuachalalate (Amphipterygium adstringens Schiede ex Schlecht). SAGARPA. INIFAP. Publicación especial No. 34. C. E. Zacatepec Mor., México. 13 p.

Solares A. F., J. Jasso-M., J. Vargas-H., M. R. Soto-H. y C. Rodríguez-F. (2006) Capacidad de regeneración en grosor y lateral en corteza de cuachalalate (Amphipterygium adstringens Schiede ex Schlect) en el Estado de Morelos. Ra Ximhai 02:481-495.

Solares A. F., J. M. P. Vázquez-A. y M. C. Gálvez-C. (2012) Canales de comercialización de la corteza de cuachalalate (Amphipterygium adstringens Schiede ex Schlecht.) en México. Revista Mexicana de Ciencias Forestales 3:29-42.

Vargas E. M., E. Castro, G. Macaya y O. J. Rocha (2003) Variación del tamaño de frutos y semillas en 38 poblaciones silvestres de Phaseolus lunatus (Fabaceae) del Valle Central de Costa Rica. Revista de Biología Tropical 51:707-724.

White P. J. (2002) Recent advances in fruit development and ripening: an overview. Journal of Experimental Botany 53:1995-2000.

Willan R. L. (1991) Guía para la Manipulación de Semillas Forestales. Centro de Semillas Forestales de DANIDA. Estudio FAO Montes. Roma. 20/2. 126 p.

Zamora R. (2002) Importancia de la heterogeneidad ambiental en la ecología de plantas carnívoras mediterráneas: implicaciones para la conservación. Revista Chilena de Historia Natural 75:17-26.

Zavala C. F. (2004) Desecación de bellotas y su relación con la viabilidad y germinación en nueve especies de encino mexicanos. Ciencia Ergo Sum 11:177-185. 